\title{
Spin-orbit-assisted electron-phonon interaction and the magnetophonon resonance in semiconductor quantum wells
}

\author{
D. S. L. Abergel ${ }^{1,2}$ and Vladimir I. Fal'ko ${ }^{1}$ \\ ${ }^{1}$ Physics Department, Lancaster University, Lancaster, LA1 4YB, United Kingdom \\ ${ }^{2}$ Department of Physics and Astronomy, University of Manitoba, Winnipeg, Canada R3T 2N2 \\ (Received 3 July 2007; revised manuscript received 27 September 2007; published 15 January 2008)
}

\begin{abstract}
We introduce a spin-orbit assisted electron-phonon (e-ph) coupling mechanism for carriers in semiconductor quantum wells and predict qualitatively the form and shape of anticrossings between the cyclotron resonance (CR) and optical (LO and TO) phonons. Since this e-ph interaction involves the electron spin it makes the predicted TO-CR anticrossing dependent on the filling factor and therefore on the spin polarization of the two-dimensional electron gas in the quantum well.
\end{abstract}

DOI: 10.1103/PhysRevB.77.035317

PACS number(s): 73.21.Fg, 71.70.Ej, 73.21.-b

\section{INTRODUCTION}

Recently, the fabrication of very thin semiconductor films with embedded quantum wells (QWs), e.g., in GaAs/AlGaAs structures, has made it possible to perform cyclotron resonance (CR) experiments in the frequency range which covers the restrahlen band in this material. ${ }^{1}$ As a result, the regime where the cyclotron transition in the two-dimensional electron gas (2DEG) is in resonance with the longitudinal (LO) and transverse (TO) optical lattice vibrations became feasible to study, subject to the coupling between these modes. This situation is known in the literature as the magnetophonon resonance. ${ }^{2}$

In the magnetophonon resonance conditions, the $\mathrm{CR}$ and the optical phonon modes may mix and form anticrossings. Resonant coupling of the optical and acoustic phonons often occurs in bulk materials via standard deformation and polarization potential interactions. ${ }^{3}$ However, the situation in quantum wells of III-V semiconductors appears to be different due to the lattice symmetry of these crystals. ${ }^{4-6}$ If the incident radiation and the magnetic field are orientated perpendicularly to the plane of the $2 \mathrm{DEG}$, there is no CR-LO phonon mixing as the in-plane motion of the electrons and the out-of-plane motion of the lattice cannot couple. Also there is no CR-TO mixing because this phonon does not carry any electric field and the deformation associated with the $q=0$ phonon does not allow for the emission or absorption of TO phonons by electrons.

In this paper, we propose a mechanism of electronphonon interaction which does permit the mixing of the CR and phonon modes. This mechanism is assisted by the spinorbit (SO) coupling in the atomic shells of the material and involves the electron spin in the interaction process. We find that in a perpendicular magnetic field, the CR will couple to the TO phonon, with the strongest coupling occurring when the electron gas is fully spin-polarized (for example, at filling factor $\nu=1)$ and reducing to zero when the electron gas is completely un-spin-polarized (e.g., at $\nu=2$ ).

We model the quantum well containing the 2DEG as a thin slab of polar semiconductor mounted on a nonpolar substrate. Electrons are confined in a QW which is embedded in a matrix of a material which has not only a different bandgap but also a nonoverlapping phonon density of states. For a thin slab there are two phonon modes polarized in the plane of the slab with the frequency $\omega_{x, y}(q \rightarrow 0)=\omega_{T O}$ and one mode polarized perpendicularly to the slab with $\omega_{z}(q \rightarrow 0)=\omega_{L O}$.

For semiconductors with a zinc-blende lattice structure we write down ${ }^{7,8}$ the SO-assisted (Rashba-type ${ }^{9}$ ) electron interaction with lattice displacements as ${ }^{10}$

$$
\mathcal{H}_{e-p h}=\frac{\alpha}{2} \boldsymbol{\sigma} \cdot(\mathbf{p} \times \mathbf{w}+\mathbf{w} \times \mathbf{p}) .
$$

This term is invariant with respect to the transformations from the symmetry group ${ }^{4}$ of the zinc-blende type lattice $T_{d}$. The vector $\mathbf{w}$ represents the relative displacement of the two sublattices with respect each other, which acts to deform the atomic bonds. This in turn changes the hopping integrals of electrons between SO-mixed orbitals and provides the mechanism for the coupling in Eq. (1), parametrized using a material-dependent phenomenological constant $\alpha$.

\section{MODE COUPLING}

For optical phonons confined to a $N$-atomic-layer thick $\mathrm{QW}$, the sublattice displacement field $\mathbf{w}(\mathbf{r})=\mathbf{w}(\mathbf{x}) \beta_{k}(z)$ represents a standing wave in the slab, ${ }^{11}$ where $\mathbf{x}$ is a two-dimensional vector in the plane of the 2DEG, $\beta_{k}(z)$ $=\sqrt{2 / N} \sin (\pi k z / N a)$, and $k$ labels the phonon standing wave mode. The second quantized phonon field operator can be written as ${ }^{12}$

$$
\mathbf{w}=\sum_{\mathbf{q}, \kappa} \sqrt{\frac{\hbar a^{2}}{2 L_{x} L_{y} m_{\kappa} \omega_{\kappa}}} e^{i \mathbf{q} \cdot \mathbf{x} / \hbar} \beta(z) \mathbf{l}_{\mathbf{q}, \kappa}\left(b_{\mathbf{q}, \kappa}+b_{-\mathbf{q}, \kappa}^{\dagger}\right),
$$

where $\kappa=x, y, z$ denotes the phonon modes, $\mathbf{l}_{\mathbf{q}, \kappa}$ is the unit polarization vector of mode $\kappa$ and $b$ and $b^{\dagger}$ stand for annihilation and creation operators of phonons. The reduced mass of the atoms in the unit cell and frequency of the phonon modes are $m_{\kappa}$ and $\omega_{\kappa}$, respectively, $a$ is the lattice constant, and $L_{x, y}$ are the lateral sample sizes.

The high magnetic field CR can be discussed in terms of electron transitions between Landau levels upon absorption of a far-infrared photon. The Landau levels involved in such transitions depend on the filling factor and the sign of the 
conduction band $g$ factor. Below we study a 2DEG with $\nu \leqslant 2$ where electrons occupy only the lowest $(n=0)$ Landau level. ${ }^{13}$ In our analysis the spin polarization of the 2DEG plays a crucial role because the electron spin is involved in the interaction. Therefore we might expect the qualitative features of the coupling to differ for $\nu=1$ (a fully spinpolarized 2DEG) and $\nu=2$ (an un-spin-polarized 2DEG). The inter-Landau level transtitions for the quantum Hall effect states at $\nu \leq 2$ of electrons can be described using the magnetoexciton operators which define coherent excitations of electrons from the $n=0$ to the $n=1$ Landau level: ${ }^{14,15}$

$$
\Psi_{\alpha, \alpha^{\prime}}^{\dagger}(\mathbf{q})=\sum_{p} \frac{e^{i p q_{y}}}{\sqrt{\mathcal{N}}} a_{1, \alpha, p}^{\dagger} a_{0, \alpha^{\prime}, p-q_{x}}
$$

The indices $\alpha, \alpha^{\prime}$ represent the spin of final and initial states, respectively, $\mathcal{N}$ is the number of single electron states in each Landau level, and $a,\left(a^{\dagger}\right)$ are single electron annihilation (creation) operators.

For $\nu=2$ the ground state consists of completely filled up-spin and down-spin levels. The low-energy excitations with $q=0$ are then the spinless (CR) mode $S$ $=\left(\Psi_{\uparrow \uparrow}+\Psi_{\downarrow \downarrow}\right) / \sqrt{2}$ which has $s_{z}=0$ and energy $\hbar \omega_{c}$ and three triplet modes which carry spin $s=1: \Psi_{\uparrow \downarrow}$ which has $s_{z}=+1$ and energy $\hbar \omega_{c}+\epsilon_{z}$ (the Zeeman energy $\epsilon_{z}=g \mu_{B} B$ can be positive or negative depending on the sign of the $g$ factor, which we do not specify); $\Psi_{1 \uparrow}$ which has $s_{z}=-1$ and energy $\hbar \omega_{c}-\epsilon_{z}$; and $T=\left(\Psi_{\uparrow \uparrow}-\Psi_{\downarrow \downarrow}\right) / \sqrt{2}$ which has $s_{z}=0$ and energy $\hbar \omega_{c}$.

When $\nu=1$ the $q=0$ modes which can be excited in the 2DEG with frequency close to $\omega_{c}$ are defined by the sign of the $g$ factor. If it is negative then the 2DEG is a fully polarized system of up-spin electrons giving rise to excitations $\Psi_{\uparrow \uparrow}$ (which corresponds to the CR and has $s_{z}=0$ and energy $\hbar \omega_{c}$ ) and $\Psi_{\downarrow \uparrow}$ (which has $s_{z}=-1$ and energy $\hbar \omega_{c}+\epsilon_{z}$ ). In this case the Zeeman energy contains a contribution from the electron-electron exchange interaction. If the $g$ factor is positive then the 2DEG is fully polarized spin-down and at $\omega \approx \omega_{c}$ the excitations are $\Psi_{\downarrow \downarrow}$ (the CR which carries $s_{z}=0$ ) and $\Psi_{\uparrow \downarrow}$ (which has $s_{z}=+1$ ). All electronic excitations have $M_{z}=+1$.

To analyze the fine structure of the CR at the CR-LO and CR-TO crossings we determine the effective coupling between electron inter-Landau level excitons and the lattice vibrations. We represent the wave functions of electrons in the QW subjected to a high magnetic field in the form $\Phi(\mathbf{r})=\psi_{n, p}\left(y / \lambda_{B}\right) \varphi_{0}(z)$ adapted to the Landau gauge with vector potential $\mathbf{A}=-B y \mathbf{l}_{x}$ where $\lambda_{B}=\sqrt{\hbar /(e B)}$ is the magnetic length. The function $\varphi_{0}(z)=\sqrt{2} / N a \sin (\pi z / N a)$ describes the form of the electron wave function in the QW and the Landau level states $\psi_{n, p}$ are related to each other by raising and lowering operators $\pi=-\lambda_{B} / \sqrt{2} \hbar\left(p_{x}-i p_{y}\right)$ and $\pi^{\dagger}=-\lambda_{B} / \sqrt{2} \hbar\left(p_{x}+i p_{y}\right)$ such that $\pi^{\dagger} \psi_{n, p}=\sqrt{n+1} \psi_{n+1, p}, \pi \psi_{n, p}$ $=\sqrt{n} \psi_{n-1, p}$, and $\pi \psi_{0, p}=0$. Since each electron in the 2DEG covers many $(N)$ atomic layers, the effective coupling of an electron is given by

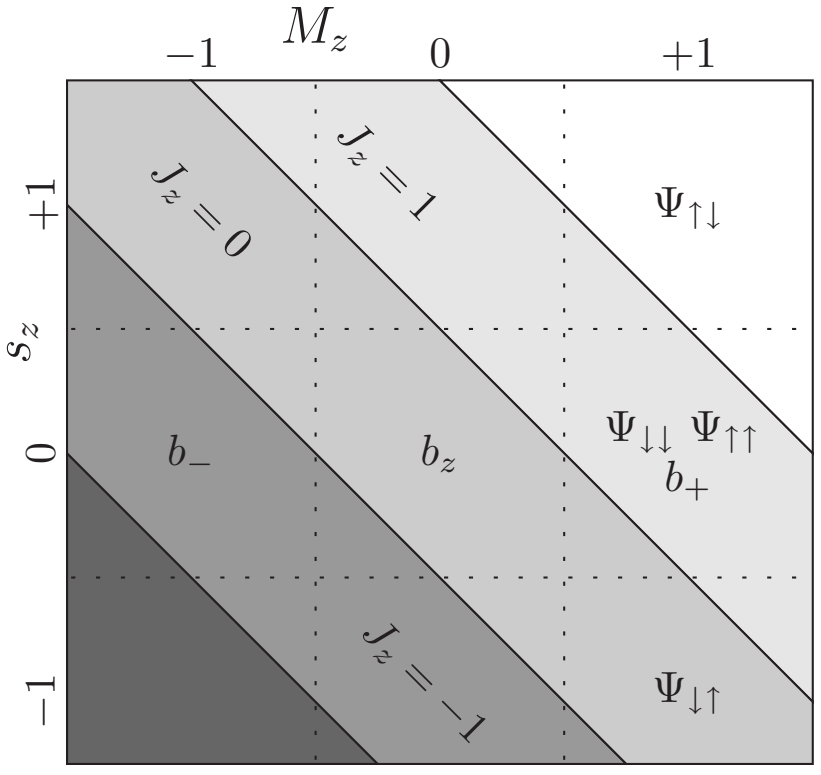

FIG. 1. This figure shows the quantum numbers (i.e., the projection of the orbital angular momentum $M_{z}$ and the spin $s_{z}$ onto the direction of the magnetic field) of each of the primary modes in the system. When the magnetic field is perpendicular to the 2DEG, coupling is allowed only between modes which conserve $J_{z}=M_{z}$ $+s_{z}$, which are shown in regions of the same color.

$$
Z_{p}=\frac{2 \pi \xi_{k} \hbar a}{\lambda_{B}} \sqrt{\frac{2 \hbar}{m_{p} \omega_{p}}},
$$

which is reduced by the factor

$$
\xi_{k}=\frac{\alpha}{2} \int_{0}^{N a} \beta_{k}(z)\left|\varphi_{0}(z)\right|^{2} d z= \begin{cases}0 & \text { even } k, \\ \frac{4 \alpha}{k\left(4-k^{2}\right) \pi} \sqrt{\frac{2}{N}} & \text { odd } k .\end{cases}
$$

The coupling is strongest with the lowest mode $k=1$ which will be the only mode we discuss in the following analysis. The subscript $p=\mathrm{LO}$, TO denotes the phonon branch.

When $\mathbf{B}=B \mathbf{l}_{z}$, the rotational symmetry about the $z$ axis of both the Hamiltonian and the interaction makes the projection $J_{z}=M_{z}+s_{z}$ of the total angular momentum carried by an excitation an exact quantum number. As a result only modes which have the same value of $J_{z}$ can mix by the interaction in Eq. (1), as illustrated in Fig. 1 where each excitation has been placed according to the $z$ projection of its orbital angular momentum and spin. The electron and phonon modes which can mix must belong to the same constant- $J_{z}$ "diagonal" highlighted using shaded stripes. To define the TO phonon modes with fixed angular momentum near the $\Gamma$ point (center) of the Brillouin zone we introduce the linear combinations of degenerate $x$ - and $y$-polarized vibrations, ${ }^{8,16}$

$$
b_{ \pm}=\left(b_{y} \pm i b_{x}\right) / \sqrt{2} \text {. }
$$

Then $b_{+}$becomes the annihilation operator for the phonon carrying $M_{z}=+1$ and $b_{-}$for the phonon with $M_{z}=-1$. For completeness we mention that the $z$-polarized vibration $b_{z}$ (with LO phonon frequency) has $M_{z}=0$ and all phonon 

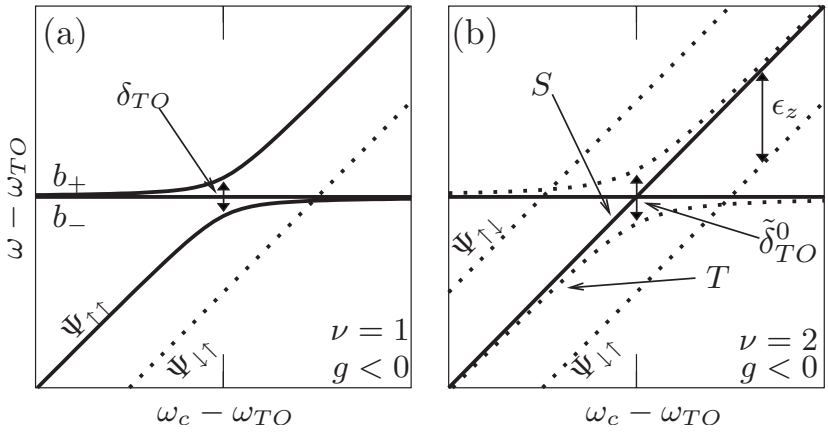

FIG. 2. Anticrossings of electronic modes and the TO phonon. Throughout the solid lines denote optically active modes (the CR and phonon modes) while dotted lines represent the optically passive spinful electronic modes. (a) When the CR crosses the TO mode at $\nu=1$ there is a splitting of magnitude $\delta_{T O}=2 Z_{T O}$. (b) When $\nu=2$ the $\mathrm{CR}$ is decoupled from the TO phonons $\left(\delta_{T O}=0\right)$, but the spinful magnetoexciton $T$ mixes with $b_{+}$and has a splitting of $\tilde{\delta}_{T O}^{0}=2 \sqrt{2} Z_{T O}$. The tick marks on the horizontal axis denote the point $\omega_{c}=\omega_{T O}$.

modes have $s=0$. For example, the selection rule for the mode mixing illustrated in Fig. 1 shows that the phonon mode $b_{-}$and the spin-flip mode $\Psi_{\uparrow \downarrow}$ cannot mix with any other modes but that $b_{+}$may couple only to $\Psi_{\uparrow \uparrow}$ or $\Psi_{\downarrow \downarrow}$.

Below we consider seperately the couplings at filling factor $\nu=1$ (for positive and negative $g$ ) and for filling factor $\nu=2$ since each of these systems has its own set of magnetoexcitons. The fine structure of the spectrum of modes $\Omega_{e p}^{ \pm}$ near a crossing can be described by the equation

$$
\hbar \Omega_{e p}^{ \pm}=\left(\hbar \omega_{e}+\hbar \omega_{p}\right) / 2 \pm \sqrt{\left(\hbar \omega_{e}-\hbar \omega_{p}\right)^{2} / 4+\gamma_{p} Z_{p}^{2}},
$$

found by diagonalizing the appropriate Hamiltonian directly. The subscripts $e$ and $p=\mathrm{LO}, \mathrm{TO}$ identify the electronic and phonon modes involved in the coupling, respectively. The numerical parameter $\gamma_{p}$ is filling-factor-dependent and should be specified for each individual pair of modes. We calculate the size of the minimum splitting as $\hbar\left(\Omega_{e p}^{+}-\Omega_{e p}^{-}\right)$ $=2 \sqrt{\gamma_{p}} Z_{p}$ exactly at the resonance and label it by $\delta_{p}$ for optically active electronic modes, $\tilde{\delta}_{p}^{0}$ for passive modes with $s_{z}=0$, and $\widetilde{\delta}_{p}^{s}$ for passive modes with $\left|s_{z}\right|=1$.

\section{A. Fully spin-polarized electron gas, $\nu=1$}

For a fully spin-polarized electron gas at filling factor $\nu$ $=1$ and $g<0$ the electrons fill the up-spin states and therefore the magnetoexcitons in this configuration ${ }^{16}$ are the optically passive $\Psi_{\downarrow \uparrow}$ and the CR mode $\Psi_{\uparrow \uparrow}$. The Hamiltonian describing possible couplings of the electron and phonon modes generated by the microscopic mechanism in Eq. (1) can be written down as
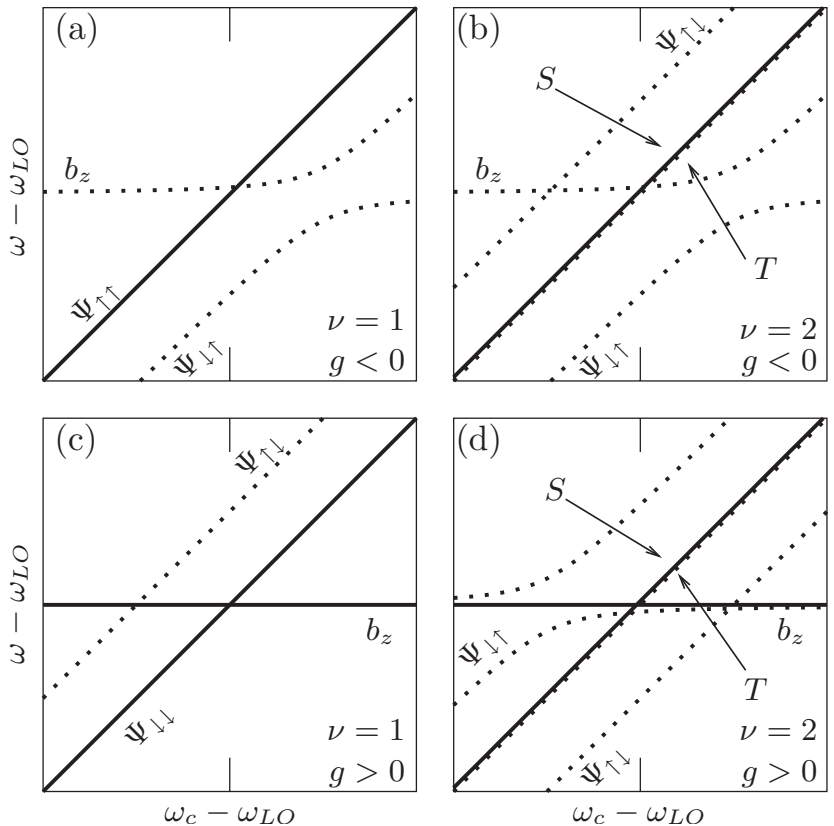

FIG. 3. Crossings in the region of the LO phonon. Solid lines denote optically active electronic modes (that is, the CR) and the phonon modes; dotted lines represent spinful electronic modes. (a) When $\nu=1$ and $g<0$ the CR is decoupled from the LO phonon, but there is a splitting of magnitude $\widetilde{\delta}_{L O}^{s}=2 \sqrt{2} Z_{L O}$ between the LO phonon and the spinful $\Psi_{\downarrow \uparrow}$ mode. (b) For $\nu=2$ and $g<0$ the CR is decoupled from the LO phonon, but the mode $\Psi_{\downarrow \downarrow}$ does split with magnitude $\widetilde{\delta}_{L O}^{s}=2 \sqrt{2} Z_{L O}$. (c) When $\nu=1$ and $g>0$ there is no coupling of any modes. (d) For $\nu=2$ and $g>0$, the coupled spinful mode splits from the LO phonon with magnitude $\widetilde{\delta}_{L O}^{s}=2 \sqrt{2} Z_{L O}$. Tick marks on the horizonal axis show where $\omega_{c}=\omega_{L O}$.

$$
\begin{aligned}
\hat{\mathcal{H}}_{\nu=1}^{g<0}= & {\left[\Psi_{\uparrow \uparrow}^{\dagger}, \hat{b}_{+}^{\dagger}, \Psi_{\downarrow \uparrow}^{\dagger}, b_{z}^{\dagger}\right] } \\
& \times\left[\begin{array}{cccc}
\hbar \omega_{c} & Z_{T O} & 0 & 0 \\
Z_{T O} & \hbar \omega_{T O} & 0 & 0 \\
0 & 0 & \hbar \omega_{c}-\left|\epsilon_{z}\right| & \sqrt{2} i Z_{L O} \\
0 & 0 & -\sqrt{2} i Z_{L O} & \hbar \omega_{L O}
\end{array}\right]\left[\begin{array}{c}
\Psi_{\uparrow \uparrow} \\
b_{+} \\
\Psi_{\downarrow \uparrow} \\
b_{z}
\end{array}\right] .
\end{aligned}
$$

Therefore at the crossing of the $\mathrm{CR}$ with the TO phonon when the electron gas is fully spin-polarized, the Hamiltonian (7) gives the coupling parameter $\gamma_{T O}=1$ which leads to the splitting $\delta_{T O}=2 Z_{T O}$, as shown in Fig. 2(a). At the crossing of the $\mathrm{CR}$ with the $\mathrm{LO}$ phonon for this filling factor and sign of the $g$ factor, the optically active mode is decoupled from the phonons (i.e., $\gamma_{L O}=0$ ), giving $\delta_{L O}=0$. In the case where $g<0$, described by the Hamiltonian in Eq. (7), the selection rules represented in Fig. 1 show that the LO phonon can couple with the optically inactive $\Psi_{\downarrow \uparrow}$ mode. The latter anticrossing is parametrized by $\gamma_{L O}=2$ which gives $\widetilde{\delta}_{L O}^{s}=2 \sqrt{2} Z_{L O}$, with the anticrossing diagram plotted in Fig. $3(\mathrm{a})$. 
When $g>0$ the ground state of the 2DEG with $\nu=1$ consists of down-spin electrons. The excitations ${ }^{16}$ from this state are $\Psi_{\downarrow \downarrow}$ and $\Psi_{\uparrow \downarrow}$ and the Hamiltonian is

$$
\begin{aligned}
\hat{\mathcal{H}}_{\nu=1}^{g>0}= & {\left[\Psi_{\downarrow \downarrow}^{\dagger}, \hat{b}_{+}^{\dagger}, \Psi_{\uparrow \downarrow}^{\dagger}, b_{z}^{\dagger}\right] } \\
& \times\left[\begin{array}{cccc}
\hbar \omega_{c} & Z_{T O} & 0 & 0 \\
Z_{T O} & \hbar \omega_{T O} & 0 & 0 \\
0 & 0 & \hbar \omega_{c}-\epsilon_{z}^{\prime} & 0 \\
0 & 0 & 0 & \hbar \omega_{L O}
\end{array}\right]\left[\begin{array}{c}
\Psi_{\downarrow \downarrow} \\
b_{+} \\
\Psi_{\uparrow \downarrow} \\
b_{z}
\end{array}\right] .
\end{aligned}
$$

Using the diagram in Fig. 1 we find that while $\Psi_{\downarrow \downarrow}$ may couple to the $b_{+}$phonon (which leads to the same splitting $\delta_{T O}=2 Z_{T O}$ as for the $g<0$ case), the conservation of $J_{z}$ forbids any coupling of $\Psi_{\uparrow \downarrow}$. This means that there is no coupling between magnetoexcitons and the $b_{z}$ phonon, so that $\widetilde{\delta}_{L O}^{s}=0$.

\section{B. Unpolarized electron gas, $\nu=2$}

In the 2DEG with filling factor $\nu=2$ the up-spin and down-spin states in the lowest Landau level are filled so that the excitations in the 2DEG with energy close to $\hbar \omega_{c}$ are ${ }^{16}$ $\Psi_{\uparrow \uparrow}, \Psi_{\uparrow \downarrow}, \Psi_{\downarrow \uparrow}$, and $\Psi_{\downarrow \downarrow}$. Figure 1 shows that linear combinations of $\Psi_{\uparrow \uparrow}$ and $\Psi_{\downarrow \downarrow}$ may couple to the $b_{+}$phonon and that $\Psi$ may couple to the $b_{z}$ phonon. The linear combinations which are relevant are the modes $S=\left(\Psi_{\uparrow \uparrow}+\Psi_{\downarrow \downarrow}\right) / \sqrt{2}$ and $T$ $=\left(\Psi_{\uparrow \uparrow}-\Psi_{\downarrow \downarrow}\right) / \sqrt{2}$. The mode $S$ is spinless and therefore corresponds to the $\mathrm{CR}$. The resulting Hamiltonian is

$$
\begin{aligned}
\hat{\mathcal{H}}_{\nu=2}= & {\left[S^{\dagger}, T^{\dagger}, b_{+}^{\dagger}, \Psi_{\downarrow \uparrow}^{\dagger}, b_{z}^{\dagger}\right] } \\
& \times\left[\begin{array}{ccccc}
\hbar \omega_{c} & 0 & 0 & 0 & 0 \\
0 & \hbar \omega_{c} & \sqrt{2} Z_{T O} & 0 & 0 \\
0 & \sqrt{2} Z_{T O} & \hbar \omega_{T O} & 0 & 0 \\
0 & 0 & 0 & \hbar \omega_{c}+\epsilon_{z} & -\sqrt{2} i Z_{L O} \\
0 & 0 & 0 & \sqrt{2} i Z_{L O} & \hbar \omega_{L O}
\end{array}\right] \\
& \times\left[\begin{array}{c}
S \\
T \\
b_{+} \\
\Psi_{\downarrow \uparrow} \\
b_{z}
\end{array}\right] .
\end{aligned}
$$

The uncoupled modes $b_{-}$and $\Psi_{\uparrow \downarrow}$ are not shown. This Hamiltonian reveals that for $\nu=2$ the CR is decoupled from the phonons at the TO resonance because the spin polariza- tion of the $\nu=2$ ground state is zero, that is $\delta_{L O}=0$. However, coupling between the spinful mode $T$ and the TO phonon, and $\Psi_{\downarrow \uparrow}$ and the LO phonon are possible and are parametrized by $\gamma_{T O}=\gamma_{L O}=2$ leading to $\widetilde{\delta}_{T O}^{0}=\widetilde{\delta}_{L O}^{0}=2 \sqrt{2} Z_{p}$. The resulting anticrossing diagrams are shown in Fig. 2(b) for the TO crossing, Fig. 3(b) for the LO crossing with $g<0$, and Fig. 3(d) for the LO crossing with $g>0$.

\section{Mode coupling in a tilted magnetic field}

If the magnetic field is slightly tilted away from the $z$ direction, e.g., as $\mathbf{B}=B_{\|} \mathbf{l}_{x}+B_{\perp} \mathbf{l}_{z}=B\left(\sin \theta \mathbf{l}_{x}+\cos \theta \mathbf{l}_{z}\right)$, then $J_{z}$ is no longer a good quantum number and additional couplings are permitted. The Landau levels in the 2DEG are defined with respect to the perpendicular component of the magnetic field and accordingly $\lambda_{B}=\sqrt{\hbar /\left(e B_{\perp}\right)}$. The coupling of the $\mathrm{CR}$ at the TO resonance shows the same qualitative features as before, with a reduced splitting value $\delta_{T O}=2 Z_{T O} \cos \theta$ for $\nu=1$ whereas it remains as $\delta_{T O}=0$ for $\nu=2$. Regarding the optically passive couplings near the TO resonance, the $s_{z}=0$ mode $T$ can couple to the $b_{+}$phonon with $\widetilde{\delta}_{T O}^{0}=2 \sqrt{2} Z_{T O} \cos \theta$, and the spinful modes $\Psi_{\uparrow \downarrow}$ and $\Psi_{\downarrow \uparrow}$ can each couple to the $b_{+}$phonon with $\widetilde{\delta}_{T O}^{s}=2 Z_{T O} \sin \theta$.

Also the CR in a tilted field can show a filling-factordependent coupling with the $b_{z}$ phonon which has the frequency $\omega_{L O}$. At $\nu=1$ the splitting is given by $\delta_{L O}=\sqrt{2} Z_{L O} \sin \theta$ and at $\nu=2$ by $\delta_{L O}=0$. When $\nu=2$ the $T$ mode can couple to the $b_{z}$ phonon with $\delta_{L O}^{0}$ $=2 Z_{L O} \sin \theta$, and the $\left|s_{z}\right|=1$ modes can couple with the $b_{z}$ phonon with $\widetilde{\delta}_{L O}^{s}=\sqrt{2} Z_{L O}(\cos \theta+1)$ for the $\Psi_{\downarrow \uparrow}$ mode and $\widetilde{\delta}_{L O}^{s}=\sqrt{2} Z_{L O}(\cos \theta-1)$ for the $\Psi_{\uparrow \downarrow}$ mode.

\section{CONCLUSIONS}

In conclusion, we showed how an e-ph coupling mechanism in III-V semiconductor quantum wells which involves the coupling of the electron spin to the lattice vibrations leads to anticrossings between the CR and TO phonon mode, and the inter-Landau-level spinful magnetoexciton with both the TO and LO phonons. The resulting anticrossings of the electronic and lattice modes depend on the polarization of the electron gas and therefore vary with the filling factor. The coupling of the $\mathrm{CR}$ with the TO phonon is strongest when the electron gas is fully spin-polarized-that is, at filling factor $\nu=1$. The effect is absent in the vicinity of $\nu=2$. Tilting of the magnetic field also generates a weak anticrossing between the CR and the LO phonon which shows the same filling factor dependence as the CR-TO mixing.

\section{ACKNOWLEDGMENTS}

We would like to acknowledge helpful discussions with G. Martinez, B. Narozhny, and M. Goerbig. This work was funded by the Lancaster Portfolio Partnership and ESFFoNE project SpiCo. 
${ }^{1}$ C. Faugeras, D. K. Maude, G. Martinez, L. B. Riga, C. Proust, K. J. Friedland, R. Hey, and K. H. Ploog, Phys. Rev. B 69, 073405 (2004).

${ }^{2}$ J. P. Maneval, A. Zylberzstejn, and H. F. Budd, Phys. Rev. Lett. 23, 848 (1969); G. Bauer and H. Kahlert, Phys. Rev. B 5, 566 (1972).

${ }^{3}$ M. S. Kushwaha, Surf. Sci. Rep. 41, 1 (2001).

${ }^{4}$ G. L. Bir and G. E. Pikus, Symmetry and Strain-Induced Effects in Semiconductors (Wiley, New York, 1974).

${ }^{5}$ C. Faugeras, G. Martinez, A. Riedel, R. Hey, K. J. Friedland, and Yu. Bychkov, Phys. Rev. Lett. 92, 107403 (2004); S. N. Klimin and J. T. Devreese, ibid. 94, 239701 (2005); C. Faugeras, G. Martinez, and Yu. Bychkov, ibid. 94, 239702 (2005).

${ }^{6}$ S. N. Klimin and J. T. Devreese, Phys. Rev. B 68, 245303 (2003); G. Q. Hai, F. M. Peeters, and J. T. Devreese, ibid. 48, 4666 (1993); 62, 10572 (2000).

${ }^{7}$ V. I. Fal'ko and B. N. Narozhny, Phys. Rev. B 74, 012501 (2006).

${ }^{8}$ M. O. Goerbig, J.-N. Fuchs, K. Kechedzhi, and V. I. Falko, Phys. Rev. Lett. 99, 087402 (2007).

${ }^{9}$ E. I. Rashba and E. Ya. Sherman, Phys. Lett. A 129, 175 (1988); Y. A. Bychkov and E. I. Rashba, Pis'ma Zh. Eksp. Teor. Fiz. 39, 66 (1984) [JETP Lett. 39, 78 (1984)].

${ }^{10}$ The second quantized form of the electron-phonon interaction Hamiltonian (1) is

$$
\begin{aligned}
\hat{\mathcal{H}}_{e-p h}= & \xi_{k} \sum_{n, n^{\prime}, p, p^{\prime}} \int d \mathbf{x} \psi_{n, p}^{*}(\mathbf{p} \times \hat{\mathbf{w}}+\hat{\mathbf{w}} \times \mathbf{p}) \psi_{n^{\prime} p^{\prime}} \\
& \times\left(a_{n, p, \uparrow}^{\dagger} a_{n, p, \downarrow}^{\dagger}\right) \boldsymbol{\sigma}\left(\begin{array}{l}
a_{n^{\prime}, p^{\prime}, \uparrow} \\
a_{n^{\prime}, p^{\prime}, \downarrow}
\end{array}\right),
\end{aligned}
$$

where $\xi$ is the matrix element of the $z$-dependent part of the Hamiltonian.

${ }^{11}$ The density of states at the TO and LO frequencies in the material which surrounds the quantum well is zero: these phonon modes are confined and we use zero boundary conditions.

${ }^{12}$ J. M. Ziman, Electrons and Phonons; the Theory of Transport Phenomena in Solids (Clarendon Press, Oxford, 1960).

${ }^{13}$ Although such a crossing requires a high magnetic field $(\sim 30 \mathrm{~T})$, the use of a broad density range $10^{11}<n_{e}$ $<10^{13} \mathrm{~cm}^{-1}$ in the recent experiments requires us to study the filling factor dependence of the anticrossing with a filling factor $\nu$ in the 2DEG as large as $\nu=2$ despite the high magnetic field.

${ }^{14}$ Yu. A. Bychkov, S. V. Iordanskii, and G. M. Eliashberg, Pis'ma Zh. Eksp. Teor. Fiz. 33, 152 (1981) [JETP Lett. 33, 143 (1981)].

${ }^{15}$ C. Kallin and B. I. Halperin, Phys. Rev. B 30, 5655 (1984).

${ }^{16}$ Note that the momentum index is missing from the creation and annihilation operators since we only study excitations with $q=0$. 